\title{
Se alleviates homocysteine-induced fibrosis in cardiac fibroblasts via downregulation of IncRNA MEG3
}

\author{
WEI LI ${ }^{1 *}$, YUANHONG LI $^{2 *}$, SHENGYU CUI $^{1}$, JIAYI LIU ${ }^{1}$, \\ LIJIAO TAN ${ }^{3}$, HAO XIA ${ }^{1}$ and CHANGJIANG ZHANG ${ }^{1,4}$ \\ ${ }^{1}$ Department of Cardiology, Renmin Hospital of Wuhan University, Cardiovascular Research Institute of \\ Wuhan University, Wuhan, Hubei 430060; ${ }^{2}$ Department of Cardiovascular Biology, The Central Hospital of \\ Enshi Autonomous Prefecture; ${ }^{3}$ Medical School of Enshi Polytechnic; ${ }^{4}$ Department of Cardiovascular \\ Biology, Minda Hospital of Hubei Minzu University, Enshi, Hubei 445000, P.R. China
}

Received March 16, 2021; Accepted August 11, 2021

DOI: $10.3892 /$ etm.2021.10704

\begin{abstract}
Selenium ( $\mathrm{Se}$ ) is considered to have antioxidant properties, which are beneficial for heart condition. Hyperhomocysteinemia (HHCY) has been suggested to potentially lead to heart failure and is characterized by cardiac fibrosis; however, investigation on the role of Se and HHCY in cardiac fibrosis is rare. Since previous studies demonstrated the important role of the long non-coding RNA maternally expressed 3 (MEG3) in some heart diseases, the present study aimed to determine how Se and MEG3 might exert regulatory effects on HCY-induced fibrosis in cardiac fibroblasts (CFs). Mouse CFs were isolated and treated with HCY and Se. The expression of $\alpha$-smooth muscle actin ( $\alpha$-SMA), collagen I and III was detected by western blotting to reflect CF fibrosis. Reverse transcription-quantitative PCR was performed to determine the expression levels of MEG3. Inflammation and oxidative stress responses were analyzed by measuring TNF- $\alpha$, IL-1 $\beta$ (ELISA) and reactive oxygen species levels (using a commercial kit), respectively. Cell Counting Kit- 8 was used to evaluate $\mathrm{CF}$ proliferation. Total and phosphorylated (p) expression of janus kinase 2 (JAK2) and signal transducer and activator of transcription 3 (STAT3) was evaluated by western blotting. CFs were transfected with adenovirus expressing
\end{abstract}

Correspondence to: Professor Hao Xia, Department of Cardiology, Renmin Hospital of Wuhan University, Cardiovascular Research Institute of Wuhan University, 238 Jiefang Road, Wuhan, Hubei 430060, P.R. China

E-mail: xiahao1966@163.com

Dr Changjiang Zhang, Department of Cardiovascular Biology, Minda Hospital of Hubei Minzu University, 2 Wufengshan Road, Enshi, Hubei 445000, P.R. China

E-mail: zcj2008@163.com

*Contributed equally

Key words: selenium, cardiac fibrosis, hyperhomocysteinemia, long non-coding RNA maternally expressed 3, cardiac fibroblasts
MEG3 short-hairpin RNA to knock down MEG3 expression. Se treatment downregulated the expression level of MEG3 in HCY-stimulated CFs, whilst inhibiting the inflammatory and oxidative stress response. Furthermore, Se inhibited the increased proliferation of CFs following HCY treatment. In addition, MEG3-knockdown in CFs could improve fibrosis caused by HCY. Furthermore, the ratios of p-JAK2/JAK2 and p-STAT3/STAT3 were decreased following treatment with Se or MEG3 silencing. Taken together, the findings from the present study suggested that Se may alleviate cardiac fibrosis by downregulating the expression of MEG3 and reducing the inflammatory and oxidative stress response in CFs. This suggests that Se may be a potential therapeutic option for treating cardiac fibrosis in the future.

\section{Introduction}

Cardiac fibrosis serves a key role in various forms of chronic heart disease, resulting in reduced tissue compliance and impaired heart function (1). At present, there is no specific therapy to treat patients with cardiac fibrosis. As closely associated to cardiac fibrosis and heart remodeling, hyperhomocysteinemia (HHCY) is generally known as a risk factor of cardiovascular diseases (2). Previous tudies have shown that HHCY can lead to myocardial interstitial and perivascular fibrosis and accelerate the progress of cardiac remodeling $(2,3)$. However, although the unfavorable effects of HHCY on cardiovascular disease are well documented, the underlying mechanism of HHCY leading to cardiac fibrosis remains unknown.

As one of the essential trace minerals, selenium (Se) has been demonstrated to be crucial for cardiovascular health (4). Se deficiency is associated with a significantly increased risk of cardiovascular morbidity and mortality (5). Previous studies have confirmed the antioxidant properties of Se, and it has been suggested that Se protection against inflammation or oxidative stress is mainly exerted through Se-dependent glutathione peroxidases and other selenoproteins $(6,7)$. Inflammation response is widely involved in most diseases, and to some extent, is a normal process of body defense against certain stimuli. However, excessive inflammation may 
lead to cell damage and even tissue necrosis (8). HHCY has been reported to serve an important role in pro-inflammatory processes in numerous diseases, including intestinal inflammation and aortic adventitial inflammation $(9,10)$, although the underlying mechanisms remain unclear. The present study aimed therefore to determine whether Se could exert an anti-inflammatory protection against HHCY-induced cardiac fibrosis in mouse cardiac fibroblasts (CFs) and the potential underlying mechanisms.

Recent studies reported that some non-coding RNAs show great potential in the regulation of cardiac fibrosis (11-13). Long non-coding RNAs (lncRNAs) are a type of none-coding RNA of $>200$ nucleotides in length, which participate in various biological behavioral processes, including the onset and development of some cardiac diseases (12-16). The lncRNA scaffold attachment factor B interacting lncRNA (SAIL) has been shown to improve cardiac fibrosis after myocardial infarction by regulating the transcription of fibrosis-related genes, such as $\alpha$-SMA, collagen I and III $(12,14)$. The lncRNA maternally expressed 3 (MEG3) has been reported to regulate cardiomyocyte proliferation and apoptosis $(15,16)$. Inhibiting the expression of MEG3 can reverse hypoxia-induced growth inhibition of heart progenitor cells (15). In addition, a previous study from $\mathrm{Wu}$ et al (17) demonstrated that MEG3 knockdown in cardiomyocytes can reduce cardiomyocyte apoptosis and is associated with an improvement of heart function post-myocardial infarction. Furthermore, Gong et al (18) attributed the beneficial effects of MEG3 silencing on suppressing cardiomyocyte apoptosis to the increased expression of microRNA-183, which mediates the inhibition of $\mathrm{p} 27$, by activating PI3K/AKT/FOXO3a signaling pathway in hypoxic $\mathrm{H} 9 \mathrm{C} 2$ cells. In addition, a previous study demonstrated the protection effects of MEG3 inhibition against endoplasmic reticulum stress-mediated cardiac apoptosis by targeting p53 protein (16). However, although MEG3 was reported to be mainly enriched in CFs, study focusing on the effects of MEG3 on CFs is rare. The present study aimed therefore to determine the effect of MEG3 on HHCY-induced fibrosis in CFs and to explore the possible links between $\mathrm{Se}$ and MEG3.

\section{Materials and methods}

Isolation and culture of mice CFs. The animal experimental protocols were approved by the Animal Care and Use Committee of Renmin Hospital of Wuhan University (approval no. WDRM-20200608). A total of 30 male C57BL/6 mice (age, 8-10 weeks; weight, 25-29 g) were purchased from the China Three Gorges University (approval no. SCXK 2017-0012; Yichang, China). The animals were maintained in specific-pathogen-free and environmentally controlled isolation conditions with free access to food and water (temperature, $20-25^{\circ} \mathrm{C}$; humidity, 45-55\%; 12-h light/dark cycle) for 1 week prior to the beginning of the study. Before collection of cardiac tissues, mice were sacrificed with pentobarbital sodium $(180 \mathrm{mg} / \mathrm{kg})$ injected intraperitoneally. Subsequently, hearts were removed quickly from the chest and washed three times in PBS containing 1\% penicillin-streptomycin solution (100X) (19). Excess tissues were removed and the majority of the left ventricle was collected for further shearing into $1 \mathrm{~mm}^{3}$ pieces with microsurgical scissors. Once broken tissue was digested by $0.05 \%$ trypsin for $3 \mathrm{~min}$ at $37^{\circ} \mathrm{C}$, before subsequent digestion three times at $37^{\circ} \mathrm{C}(\sim 3 \mathrm{~min}$ each time) was performed in DMEM/F12 medium (Gibco; Thermo Fisher Scientific, Inc.) containing $0.018 \%$ collagenase II (cat. no. 2275 ; Biofroxx; neoFroxx $\mathrm{GmbH}$ ). After placing at room temperature for $1 \mathrm{~min}$, the supernatant was collected and neutralized in DMEM/F12 medium containing 10\% FBS (TICO Europe) after each digestion. Neutralized supernatant was centrifuged at $1,000 \mathrm{x} \mathrm{g}$ for $9 \mathrm{~min}$ at $4^{\circ} \mathrm{C}$ to collect the cell pellet, which was further resuspended in DMEM/F12 medium containing $10 \%$ FBS and seeded in a six-well plate. CFs were obtained after $1 \mathrm{~h}$ of adherence whereas the supernatant cells that had not adhered were discarded. CFs were cultured in DMEM/F12 medium containing $10 \% \mathrm{FBS}$ at $37^{\circ} \mathrm{C}$ for $24 \mathrm{~h}$. CFs at passage two were used for subsequent experiments and were randomly divided into six groups as follows: i) Control group (Ctrl); ii) Se group, only treated with $100 \mathrm{nM}$ sodium selenite (Sigma-Aldrich; Merck KGaA; cat. no. S5261); iii) HCY (200 $\mu \mathrm{M})$-stimulated group; iv) HCY + Se group (co-treated with HCY and Se); v) HCY + adenovirus (Ad)-shMEG3 group (HCY + Ad-shMEG3); and vi) HCY + Ad-scramble group $(\mathrm{HCY}+\mathrm{Ad}-\mathrm{scr})$. CFs in six-well plates were transfected with MOI of 100 for $12 \mathrm{~h}$ at $37^{\circ} \mathrm{C}$ before subsequent drug treatment. The CFs were treated with Se and HCY at the same time at $37^{\circ} \mathrm{C}$ for $24 \mathrm{~h}$. Adenoviruses (constructed by GV119 plasmid vector) expressing short hairpin RNA (shRNA) against MEG3 (5'-ACCCTCCTGGATTAGGCCAAA-3') and negative control shRNA (5'-TTCTCCGAACGTGTCACGT-3') were generated by Shanghai Genechem Co., Ltd.

Cell proliferation assessment. Cell Counting Kit-8 (CCK-8; Dojindo Molecular Technologies, Inc.; cat. no. CK04) was used to evaluate CF proliferation. Briefly, CFs were seeded in a 96-well plate at the density of 5,000 cells/well and were treated with $100 \mathrm{nM}$ Se and $200 \mu \mathrm{M} \mathrm{HCY}$ at $37^{\circ} \mathrm{C}$ for $24 \mathrm{~h}$. Subsequently, $10 \mu 1$ CCK- 8 reagent was added to each well, and the cells were incubated at $37^{\circ} \mathrm{C}$ for $90 \mathrm{~min}$. The absorbance was read at a wavelength of $450 \mathrm{~nm}$ using a microplate spectrophotometer.

Detection of reactive oxygen species (ROS) production. Dihydroethidium (DHE; Beyotime Institute of Biotechnology; cat. no. S0063) staining was used to detect the cellular ROS production. DHE, after entering living cells, is dehydrogenated by intracellular superoxide anions to produce ethidium, which then binds to RNA or DNA to produce red fluorescence. The stronger the red fluorescence, the higher levels of intracellular superoxide production. In the present study, CFs were first seeded into 24-well plates at a density of $1 \times 10^{5}$ cells/well. DHE $(10 \mu \mathrm{M})$ was then added to CFs and incubated at $37^{\circ} \mathrm{C}$ for $30 \mathrm{~min}$ before treated being with $100 \mathrm{nM}$ Se and $200 \mu \mathrm{M}$ $\mathrm{HCY}$ at $37^{\circ} \mathrm{C}$ for $24 \mathrm{~h}$. Cells were washed with PBS and the fluorescence was observed under a fluorescence microscope using x100 magnification.

ELISA assays. Cell culture supernatant from six-well plates in each group was collected and the levels of tumor necrosis factor- $\alpha$ (TNF- $\alpha$ : ELK Biotechnology, Co., Ltd.; cat. no. ELK1395) and interleukin-1 $\beta$ (IL-1 $\beta$; ELK Biotechnology, 
Co., Ltd.; cat. no. ELK1271) were evaluated using ELISA kits according to the manufacturer's protocols.

SOD and MDA detection. First, CFs in six-well plate were lysed by RIPA lysis buffer (Beyotime Institute of Biotechnology; cat. no. $\mathrm{P} 0013 \mathrm{~B}$ ) at $4^{\circ} \mathrm{C}$. The lysate of each group was then collected, followed by centrifugation at $2,000 \mathrm{xg}$ at $4^{\circ} \mathrm{C}$ for $10 \mathrm{~min}$. SOD kit (cat. no. A001-3; Nanjing Jiancheng Bioengineering Institute) and MDA kit (cat. no. A003-1; Nanjing Jiancheng Bioengineering Institute) were used to evaluate the levels of SOD and MDA, respectively, according to the manufacturer's instruction.

Reverse transcription quantitative (RT-q)PCR. Total RNA was extracted from CFs using RNAiso Plus reagent (Takara Bio,Inc.; cat. no. 9108) and dissolved into diethyl pyrocarbonate-treated water. After testing the concentration of RNA, SweScript RT First Strand ${ }^{\circledR}$ cDNA Synthesis Kit (Wuhan Servicebio Technology Co., Ltd.; cat. no. G3330-50) was used to reverse transcribe RNA into cDNA according to the manufacturer's instructions. The following thermocycling conditions were used for PCR: Initial denaturation at $95^{\circ} \mathrm{C}$ for $30 \mathrm{sec}$, followed by 40 cycles of $95^{\circ} \mathrm{C}$ for $15 \mathrm{sec}, 60^{\circ} \mathrm{C}$ for $10 \mathrm{sec}$ and $72^{\circ} \mathrm{C}$ for $30 \mathrm{sec}$. The reactions were conducted in an ABI VIIa7 system (Applied Biosystems; Thermo Fisher Scientific, Inc.) using a 2x SYBR Green qPCR Master Mix (Low ROX; Wuhan Servicebio Technology Co., Ltd.; cat. no. G3320-05). The sequences of the primers were as follows: MEG3 forward, 3'-GCTCATCTTATT CTGGGCACCT-5' and reverse, 3'-TCGTGGACATTCCTC TTCCG-5'; and GAPDH forward, 3'-CCTCGTCCCGTAGAC AAAATG-5' and reverse, 3'-TGAGGTCAATGAAGGGGT CGT-5'. The $2^{-\Delta \Delta C q}$ method was used to analyze the result of qPCR (20).

Western blotting. Total protein was extracted from CFs lysed by RIPA lysis buffer (Beyotime Institute of Biotechnology; cat. no. P0013B) at $4^{\circ} \mathrm{C}$. Protein concentration was determined using a bicinchoninic acid protein assay kit (Beyotime Institute of Biotechnology; cat. no. P0012S). Proteins were separated by $10 \%$ SDS-PAGE ( $25 \mu \mathrm{g}$ per lane) and were transferred onto PVDF membranes. Membranes were blocked in 5\% non-fat milk at room temperature for $2 \mathrm{~h}$, followed by incubation with primary antibodies at $4^{\circ} \mathrm{C}$ overnight. The next day, membranes were washed three times with TBST ( $20 \mathrm{mM}$ Tris, $137 \mathrm{mM} \mathrm{NaCl}, 0.1 \%$ Tween) and were incubated with secondary antibodies for $1 \mathrm{~h}$ at room temperature. Membranes were washed three times with TBST and protein bands were visualized using enhanced chemiluminescence reagent (Beyotime Institute of Biotechnology; cat. no. P0018FM). The relative protein expression levels were normalized to endogenous control GAPDH using AlphaEaseFC software (FluorChem8900; Alpha Innotech Corporation; ProteinSimple). The present study used primary antibodies against GAPDH (Abcam; cat. no. ab37168; 1:10,000), collagen I (Abcam; cat. no. ab34710; 1:1,000), collagen III (Abcam; cat. no. ab7778; 1:1,000), $\alpha$-SMA (Abcam; cat. no. ab32575; 1:1,000), JAK2 (Abcam; cat. no. ab108596; 1:1,000), phosphorylated (p)-JAK2 (Cell Signaling Technology, Inc.; cat. no. 3230; 1:1,000), STAT3 (Abcam; cat. no. ab68153; 1:1,000) and p-STAT3 (Abcam; cat. no. ab76315; 1:1,000). The secondary antibody used in the present study was the horseradish peroxidase-conjugated goat anti rabbit antibody (ASPEN; cat. no. AS1107; 1:10,000).

Statistical analysis. Statistical analysis was performed using SPSS software (version 19.0; IBM Corp). All data were presented as the means \pm standard deviation. Comparisons between two groups were evaluated using unpaired two-tailed Student's t-test. Multiple comparisons were performed using one-way ANOVA followed by Bonferroni's post hoc test. $\mathrm{P}<0.05$ was considered to indicate a statistically significant difference.

\section{Results}

Se alleviates fibrosis induced by $\mathrm{HCY}$. The occurrence of cardiac fibrosis is closely related to the activation and proliferation of cardiac fibroblasts. As presented in Fig. 1A, when HCY concentration was $<200 \mu \mathrm{M}, \mathrm{HCY}$ ability to stimulate the proliferation of CFs was null, therefore the $200 \mu \mathrm{M}$ concentration was selected for subsequent experiment. To clarify the effect of Se on cardiac fibrosis, we first examined the effect of Se on CF proliferation. Different concentrations of $\mathrm{Se}$ were used and the results demonstrated that $100 \mathrm{nM}$ Se had a significant inhibitory effect on CF proliferation following treatment with HCY, and $200 \mathrm{nM}$ Se resulted in significant cytotoxicity (Fig. 1B). Furthermore, $100 \mathrm{nM}$ Se showed a significant inhibitory effect on the expression of the fibrosis related proteins $\alpha$-SMA, collagen I and III following treatment with HCY (Fig. 1C). These results demonstrate that Se may effectively improve HCY-induced CF fibrosis.

Se downregulates MEG3 and MEG3 knockdown decreases $\mathrm{HCY}$-induced fibrosis. Since MEG3 has been reported to serve a role in cardiac fibrosis (21), the expression of MEG3 was determined in different experimental groups. As presented in Fig. 2A, HCY elevated the expression level of MEG3, whereas Se treatment reversed this increase. To verify that the regulation of Se on fibrosis was MEG3-independent, an MEG3-knockdown adenovirus (Ad-shMEG3) was used to infect CFs (Fig. 2B) and the impact of MEG3 knockdown on HCY-induced CF fibrosis was explored. Consistent with the effect of Se, MEG3 knockdown significantly inhibited the increased proliferation of CFs induced by HCY (Fig. 2C). In addition, the expression of $\alpha$-SMA, collagen I and III was significantly decreased in CFs infected by Ad-shMEG3 compared with the Ad-scramble group (Fig. 2D and E). These results indicated that Se could alleviate CF fibrosis through partly downregulating MEG3.

Se or MEG3 knockdown decreases HCY-induced increase in oxidative stress. Since inflammation or oxidative stress are widely involved in progression of cardiac fibrosis (22-24), we investigated the production of ROS in the experimental groups. The results demonstrated that Se significantly inhibited the accumulation of ROS in CFs following treatment with HCY (Fig. 3A). Furthermore, Se significantly increased SOD activity and decreased MDA contents in HCY-treated CFs (Fig. 3B and C). In addition, MEG knockdown had the same anti-oxidative stress effect (Fig. 3D and F). These findings 
A

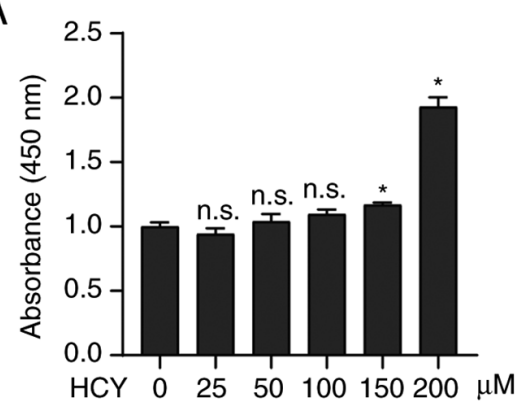

D

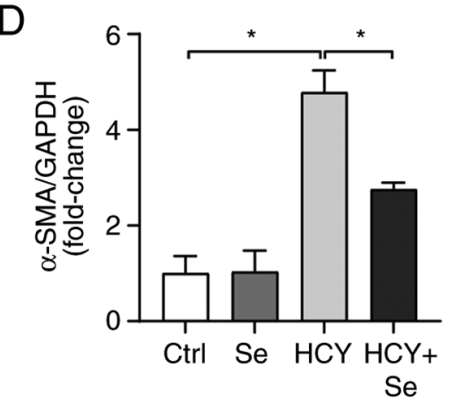

B
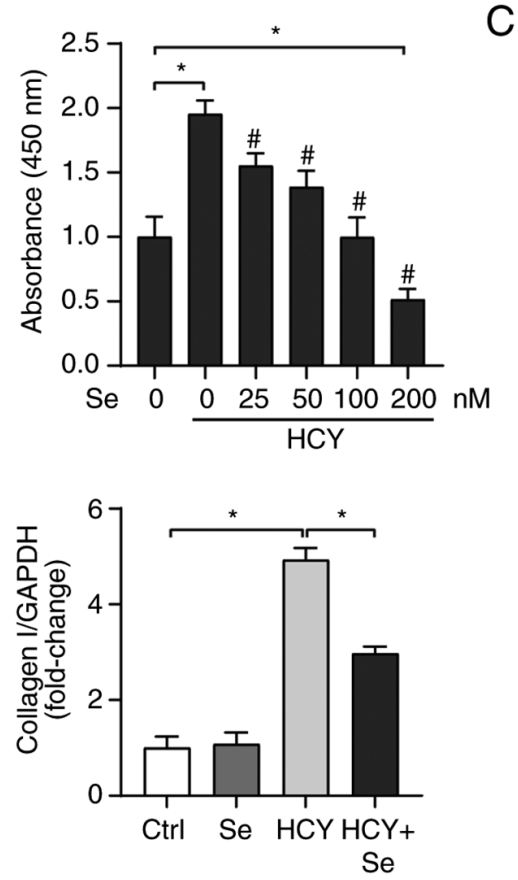

C Ctrl Se HCY HCY+Se
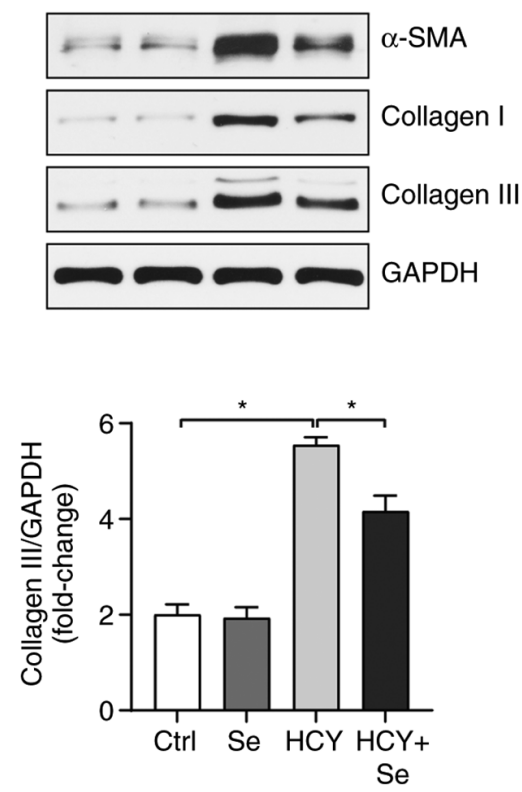

Figure 1. Se alleviates fibrosis induced by HCY. (A) Results from CCK-8 assay in CFs treated with the indicated concentrations of HCY (n=6). (B) Results from CCK-8 assay in CFs treated with HCY and the indicated concentrations of Se (n=6). (C) Expression of $\alpha$-SMA, collagen I and III assessed by western blotting in CFs stimulated with the indicated treatments. (D) Relative quantitative results of protein levels ( $\mathrm{n}=3$ ). ${ }^{*} \mathrm{P}<0.05$; ${ }^{*} \mathrm{P}<0.05$. CCK-8, Cell Counting Kit-8; CFs, cardiac fibroblasts; HCY, homocysteine; $\alpha$-SMA, $\alpha$-smooth muscle actin; NS, non-significant; Se, selenium; Ctrl, control; MEG3, maternally expressed gene 3 .
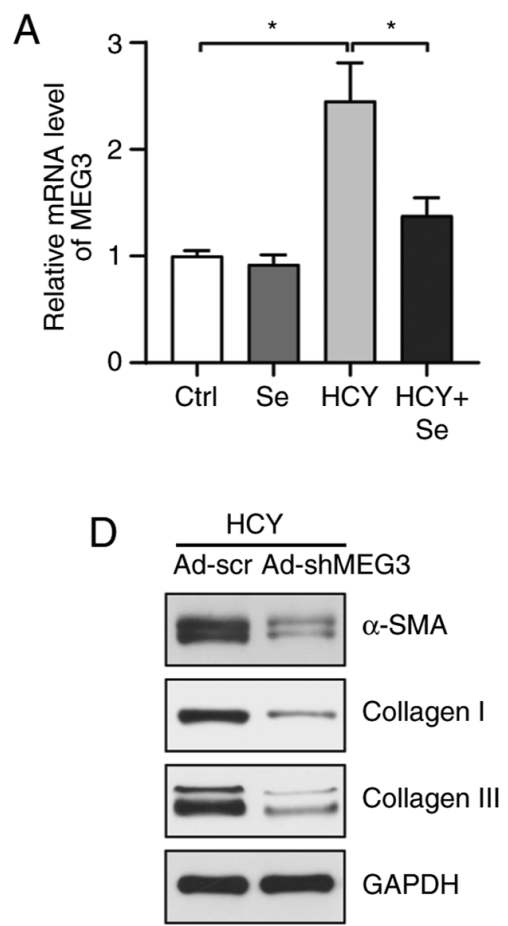

B

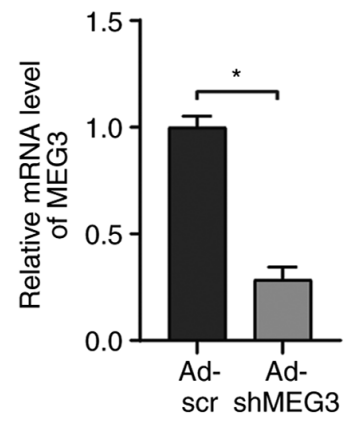

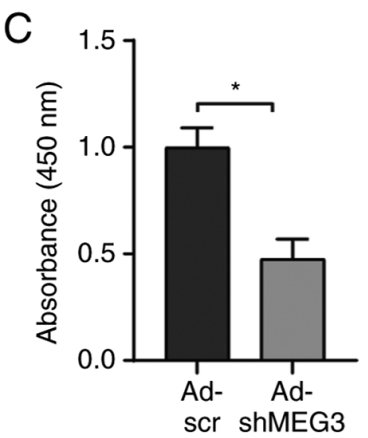

Ad-scr

$\mathrm{E}$

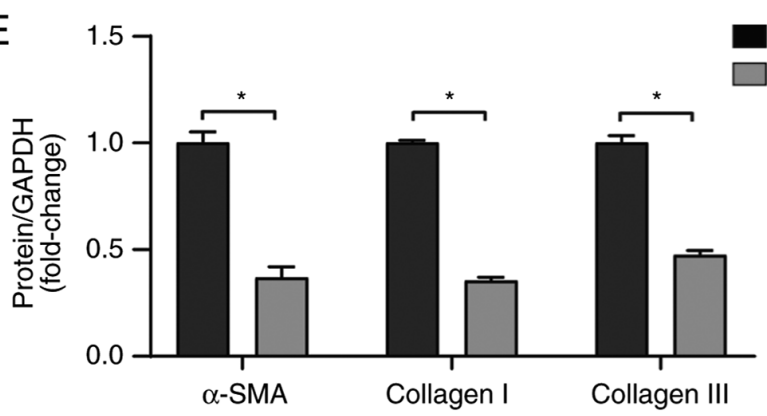

Figure 2. Se reverses HCY-induced fibrosis by downregulating the expression of MEG3. (A) Relative mRNA levels of MEG3 in CFs treated by Se and HCY for $24 \mathrm{~h}(\mathrm{n}=3)$. (B) Relative mRNA levels of MEG3 in CFs infected with Ad-scr or Ad-shMEG3 (n=3). (C) Results from Cell Counting Kit-8 assay in CFs infected with Ad-scr or Ad-shMEG3 (n=6). (D) Expression of $\alpha$-SMA, collagen I and III assessed by western blotting in CFs infected with indicated adenovirus and treated with HCY. (E) Relative quantitative results of protein levels ( $\mathrm{n}=3$ ). ${ }^{*} \mathrm{P}<0.05$. CFs, cardiac fibroblasts; HCY, homocysteine; $\alpha$-SMA, $\alpha$-smooth muscle actin; Se, selenium; Ctrl, control; Ad, adenovirus; sh, short hairpin; MEG3, maternally expressed gene 3.

suggested that the anti-oxidative effects of Se may be mediated by MEG3 regulation.
Se or MEG3 knockdown decreases the pro-inflammatory cytokines secretion. The effect of Se on inflammation was 
A

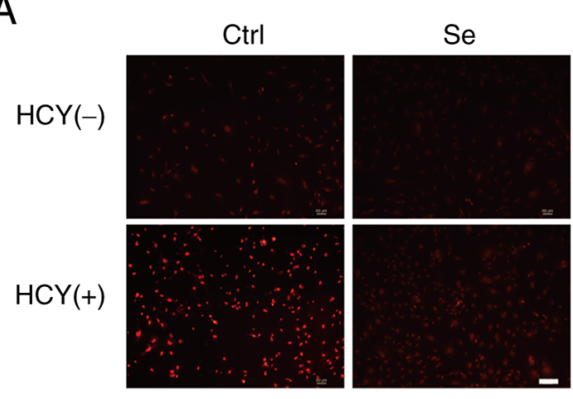

D

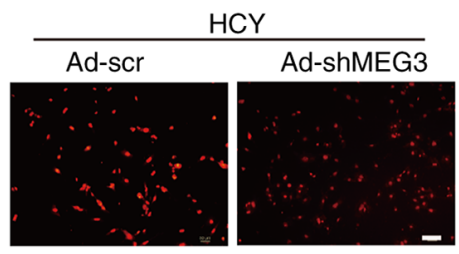

B

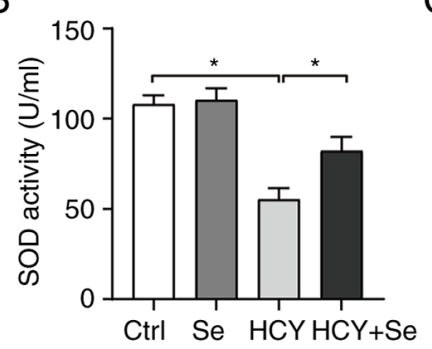

E

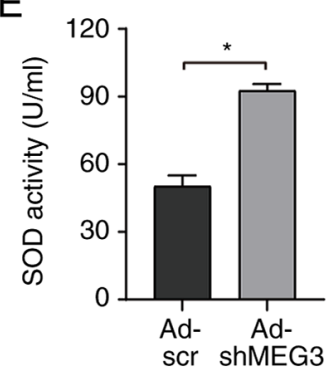

C

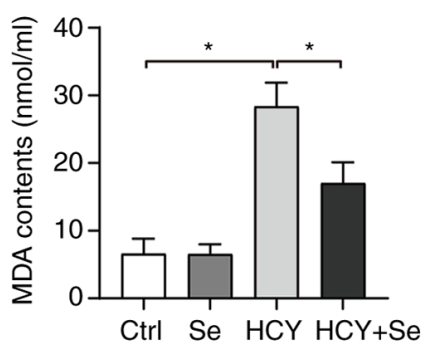

$\mathrm{F}$

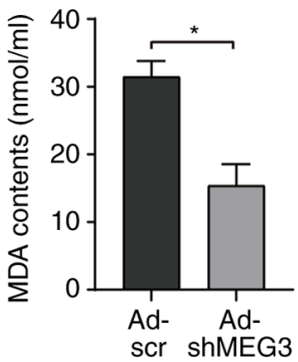

Figure 3. Se or MEG3 knockdown decreases HCY-induced oxidative stress. (A) Representative images of dihydroethidium staining in CFs stimulated with the indicated treatments. Scale bar, $100 \mu \mathrm{m}$. (B and C) (B) SOD activity and (C) MDA content in CFs stimulated with the indicated treatments ( $\mathrm{n}=3$ ). (D) Representative images of dihydroethidium staining in CFs infected with Ad-scr or Ad-shMEG3. Scale bar, $100 \mu \mathrm{m}$. (E and F) (E) SOD activity and (F) MDA content in CFs infected with Ad-scr or Ad-shMEG3 ( $\mathrm{n}=3$ ). ${ }^{*} \mathrm{P}<0.05$. CFs, cardiac fibroblasts; HCY, homocysteine; SOD, superoxide dismutase; MDA, malondialdehyde; Se, selenium; Ctrl, control; Ad, adenovirus; sh, short hairpin; MEG3, maternally expressed gene 3.

A

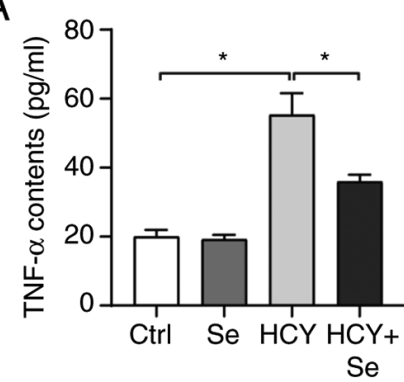

$B$

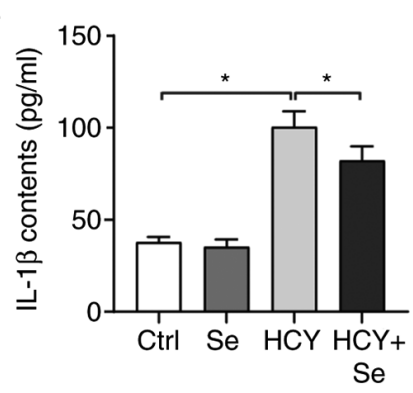

C

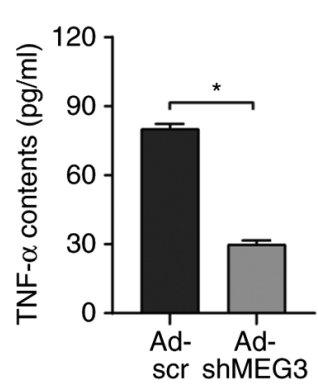

$\mathrm{D}$

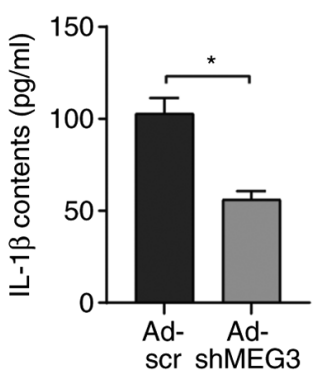

Figure 4. Se or MEG3 knockdown decreases CF pro-inflammatory cytokines secretion. (A and B) Pro-inflammatory cytokines (A) TNF- $\alpha$ and (B) IL-1 $\beta$ contents in CFs stimulated with indicated treatment were evaluated using ELISA assays ( $\mathrm{n}=3$ ). (C and D) Pro-inflammatory cytokines (C) TNF- $\alpha$ and (D) IL-1 $\beta$ contents in CFs infected with Ad-scr or Ad-shMEG3 ( $\mathrm{n}=3$ ). ${ }^{*} \mathrm{P}<0.05$. CFs, cardiac fibroblasts; TNF- $\alpha$, tumor necrosis factor- $\alpha$; IL-1 $\beta$, interleukin-1 $\beta$; Se, selenium; Ctrl, control; Ad, adenovirus; sh, short hairpin; MEG3, maternally expressed gene 3.

determined in the present study. The results from ELISA demonstrated that HCY-induced increase in TNF- $\alpha$ and IL-1 $\beta$ secretion was reversed following treatment with Se compared with control group (Fig. 4A and B). Furthermore, MEG3 knockdown alleviated $\mathrm{HCY}$-induced inflammation in $\mathrm{CFs}$ (Fig. 4C and D). These results suggested that Se may alleviate the inflammatory response caused by HCY tby regulating the expression of MEG3.

Activation of JAK2/STAT3 is mitigated following Se treatment or MEG3 knockdown. Increasing evidence suggests that JAK2/STAT3 pathway is activated during the progress of fibrosis $(25,26)$. The phosphorylation levels of JAK2 and STAT3 were therefore determined in the present study. As presented in Fig. 5, the activation of JAK2 and STAT3 was amplified in CFs following HCY treatment. Conversely, treatment with Se or MEG3 knockdown significantly decreased
p-JAK2 and p-STAT3 expression. These findings suggested that the inhibitory effect of Se on inflammation and oxidative stress may be mediated by a negative regulation of JAK2/STAT3 pathway activation.

\section{Discussion}

Cardiovascular diseases represent the leading causes of mortality worldwide (27). Almost all cardiovascular diseases are related to the pathological myocardial remodeling process that is characterized by tissue fibrosis (28-30). It is therefore crucial to clarify the specific molecular mechanism of cardiac fibrosis and to determine some targets for the treatment of cardiovascular diseases. HHCY is characterized by an abnormally high level of homocysteine in the blood, which has been reported as an independent risk factor for several cardiovascular diseases $(31,32)$. Previous studies have reported that HHCY 


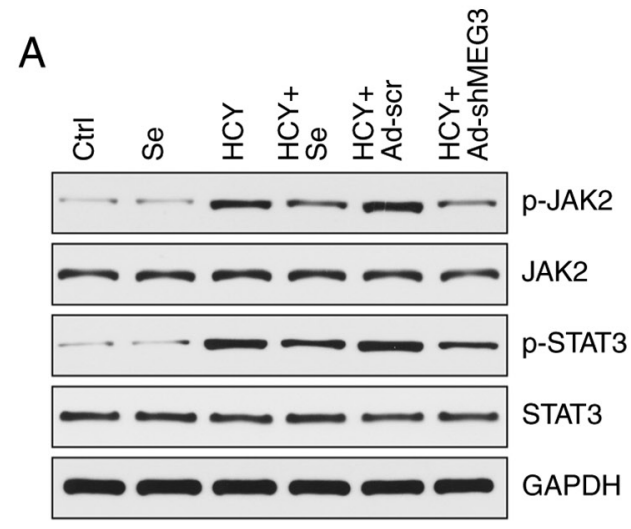

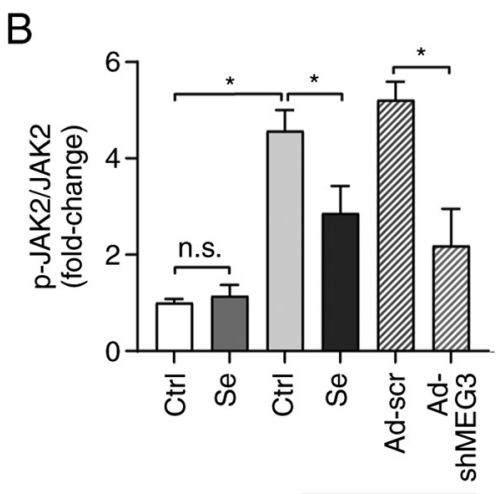

$\mathrm{HCY}$

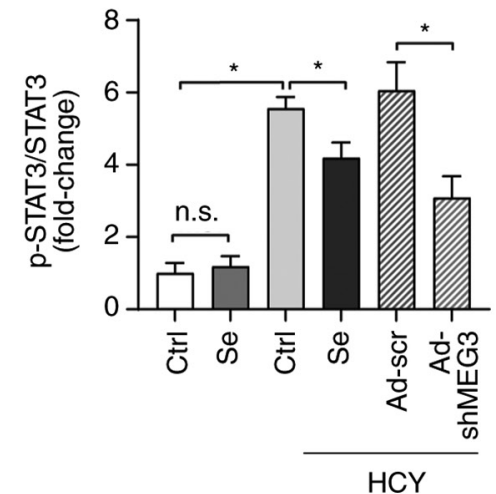

Figure 5. Activation of JAK2/STAT3 is mitigated by Se intervention or MEG3 knockdown. (A) Expression of p-JAK2, JAK2, p-STAT3 and STAT3 was evaluated by western blotting in CFs. (B) Relative quantitative results of protein levels ( $\mathrm{n}=3$ ). * $\mathrm{P}<0.05$. HCY, homocysteine; p, phosphorylated; JAK2, janus kinase 2; STAT3, signal transducer and activator of transcription 3; CFs, cardiac fibroblasts; Se, selenium; Ctrl, control; Ad, adenovirus; sh, short hairpin; MEG3, maternally expressed gene 3; NS, non-significant.

accelerates the pathological cardiac remodeling by promoting myocardial interstitial and perivascular fibrosis $(2,33)$. During pathological cardiac remodeling and cardiac fibrosis, CFs activate and secrete various matrix protein and proinflammatory cytokines including $\alpha$-SMA, collagen I and III, which can promote CF migration and proliferation (34). In the present study, we demonstrated that the secretion of the pro-inflammatory cytokines TNF- $\alpha$ and IL- $1 \beta$ and the expression of the fibrosis related genes $\alpha$-SMA, collagen I and III were significantly increased in HCY-treated CFs. Furthermore, HCY significantly promoted the proliferation of CFs. These results indicated that the effect of HHCY on cardiac fibrosis may be mediated by the promotion of $\mathrm{CF}$ proliferation and inflammation activation.

Se is an important trace element with good antioxidant effect. Se is an essential micronutrient element found in mammals and an important antioxidant of food origin $(35,36)$. Se exists in various selenoproteins and selenate in the form of selenocysteine, which serves an essential role in various diseases through inhibiting oxidative stress and inflammatory reaction (37). Previous studies have demonstrated that Se can protect cells against oxidative damage and endoplasmic reticulum stress-induced apoptosis, and can serve an important role in the reverse transport pathway during endoplasmic reticulum-related protein degradation (38). In addition, increased inflammation and oxidative stress are involved in the occurrence and development of most cardiovascular diseases (4). Previous studies have reported that $\mathrm{Se}$ alleviates the pathological progress of various cardiovascular diseases, such as heart failure and atherosclerosis, by negatively regulating inflammation and oxidative stress $(39,40)$. However, there are only a few studies about the effect of selenium on cardiac fibrosis. The present study demonstrated that Se could effectively alleviate HCY-induced cardiac fibrosis in CFs. In addition, Se significantly inhibited the activation and proliferation of $\mathrm{CFs}$, and suppressed the secretion of proinflammatory cytokines and ROS. Regarding the mechanism, Se played an anti-fibrotic effect through downregulating of MEG3. MEG3 is a 1ncRNA that possesses multiple biological functions and plays an important role in numerous diseases. Previous studies have demonstrated that MEG3 plays essential roles in cardiovascular diseases, such as inducing cardiomyocyte apoptosis, contributing to myocardial ischemia-reperfusion injury and regulating cardiac remodeling in cardiac hypertrophy (41-43). In addition, it has been reported that knocking down MEG3 expression can prevent the induction of myocardial matrix metalloproteinase-2, reduce myocardial fibrosis and improve diastolic function following transverse aortic coarctation in mice (21). The present study demonstrated that MEG3 knockdown can reduce HCY-induced CF inflammation, and that Se can effectively reduce HCY-induced inflammation by regulating the expression of MEG3.

As the most conservative members of the JAK/STAT pathway family, JAK2/STAT3 are closely related to the pathogenesis, prevention and treatment of a various types of disease, especially heart diseases such as myocardial hypertrophy, myocardial ischemia, heart failure, ischemic preconditioning and ischemia-reperfusion (44-46). A previous study demonstrated that Se deficiency in cardiomyocytes can lead to a decrease in the expression of potassium channels, mitochondrial STAT3 activity and mitochondrial function, thus promoting cardiomyocyte apoptosis (47). We therefore speculated that the effect of Se on cardiac fibrosis may be mediated by the JAK2/STAT3 pathway. The present study showed that Se or MEG3 knockdown can significantly inhibit the activation of JAK2/STAT3 signal pathway induced by HCY.

JAK2/STAT3 signal pathway is one of the important pathways of cell signal transduction, which plays an important role in cell growth, activation, differentiation and apoptosis. The main substrate of JAK kinase is a cytokine receptor, which binds to ligand in the form of dimer or monomer. However, JAK kinase has been known to activate STAT family members. Once STAT factors are phosphorylated by JAKs, they become homodimers or heterodimers, which are transferred to the nucleus, where they show activation or inhibition of transcription. It has been reported that STAT1 and STAT3 members can be activated by JAK2 in the absence of new protein synthesis and hydrogen peroxide treatment (48). At present, some studies have shown that collagen synthesis in cardiac fibroblasts can be stimulated by regulating JAK/STAT pathway, which is closely related 
to the pathological changes of cardiac fibrosis $(49,50)$. In addition, a previous study reported that hydrogen sulfide can alleviate cardiac fibrosis in diabetic rats by downregulating JAK/STAT pathway to inhibit oxidative stress and ER stress, inflammatory response and apoptosis (51). The results from the present study demonstrated that the pro-inflammatory and oxidative stress responses of cardiac fibroblasts induced by HCY was activated, which were inhibited by Se. These findings suggested that Se may not only have and antioxidant effect, but may also regulate JAK/STAT signal pathway. Since JAK2/STAT3 signal pathway can affect oxidative stress and myocardial fibrosis, and because oxidative stress leads to myocardial fibrosis and myocardial remodeling in mice $(23,24)$, we speculated that Se could inhibit cardiomyocyte apoptosis and improve myocardial fibrosis by inhibiting the activation of JAK2/STAT3 signal pathway.

In summary, the present study demonstrated that Se can inhibit the expression of MEG3 and negatively regulate the activation of JAK2/STAT3 pathway, thus effectively reducing inflammation and oxidative stress caused by HCY. These results may provide promising targets for the prevention and treatment of cardiac fibrosis.

\section{Acknowledgements}

Not applicable.

\section{Funding}

This work was supported by the Hubei Province Health and Family Planning Scientific Research Project (grant no. WJ2015MA021).

\section{Availability of data and materials}

The datasets used and/or analyzed during the current study are available from the corresponding author on reasonable request.

\section{Authors' contributions}

WL and YL performed the experiments and participated in drafting the manuscript. SC, JL and LT performed data analysis. HX and CZ designed and supervised the studies. WL and HX confirmed the authenticity of all the raw data. All authors read and approved the final manuscript.

\section{Ethics approval and consent to participate}

All animal experimental protocols were approved by the Animal Care and Use Committee of Renmin Hospital of Wuhan University (approval no. WDRM-20200608).

\section{Patient consent for publication}

Not applicable.

\section{Competing interests}

The authors declare that they have no competing interests.

\section{References}

1. Bacmeister L, Schwarzl M, Warnke S, Stoffers B, Blankenberg S, Westermann D and Lindner D: Inflammation and fibrosis in murine models of heart failure. Basic Res Cardiol 114: 19, 2019.

2. Herrmann M, Taban-Shomal O, Hübner U, Böhm M and Herrmann W: A review of homocysteine and heart failure. Eur J Heart Fail 8: 571-576, 2006.

3. Zhao Q, Song W, Huang J, Wang D and Xu C: Metformin decreased myocardial fibrosis and apoptosis in hyperhomocysteinemia-induced cardiac hypertrophy. Curr Res Transl Med 69: 103270, 2021.

4. Triposkiadis F, Xanthopoulos A and Butler J: Cardiovascular aging and heart failure: JACC review topic of the week. J Am Coll Cardiol 74: 804-813, 2019.

5. Schomburg L, Orho-Melander M, Struck J, Bergmann A and Melander O: Selenoprotein-P deficiency predicts cardiovascular disease and death. Nutrients 11: 1852, 2019.

6. Huang JQ, Zhou JC, Wu YY, Ren FZ and Lei XG: Role of glutathione peroxidase 1 in glucose and lipid metabolism-related diseases. Free Radic Biol Med 127: 108-115, 2018.

7. Hariharan S and Dharmaraj S: Selenium and selenoproteins: It's role in regulation of inflammation. Inflammopharmacology 28 : 667-695, 2020.

8. Liu X, Xia S, Zhang Z, Wu H and Lieberman J: Channelling inflammation: Gasdermins in physiology and disease. Nat Rev Drug Discov 20: 384-405, 2021.

9. Zhu S, Li J, Bing Y, Yan W, Zhu Y, Xia B and Chen M: Diet-induced hyperhomocysteinaemia increases intestinal inflammation in an animal model of colitis. J Crohns Colitis 9: 708-719, 2015.

10. Liu Z, Luo H, Zhang L, Huang Y, Liu B, Ma K, Feng J, Xie J, Zheng $\mathrm{J}, \mathrm{Hu} \mathrm{J}$, et al: Hyperhomocysteinemia exaggerates adventitial inflammation and angiotensin II-induced abdominal aortic aneurysm in mice. Circ Res 111: 1261-1273, 2012.

11. Li Y, Duan JZ, He Q and Wang CQ: miR-155 modulates high glucose-induced cardiac fibrosis via the Nrf2/HO-1 signaling pathway. Mol Med Rep 22: 4003-4016, 2020.

12. Luo S, Zhang M, Wu H, Ding X, Li D, Dong X, Hu X, Su S, Shang W, Wu J, et al: SAIL: A new conserved anti-fibrotic IncRNA in the heart. Basic Res Cardiol 116: 15, 2021.

13. Zhang F, Fu X, Kataoka M, Liu N, Wang Y, Gao F, Liang T, Dong X, Pei J, Hu X, et al: Long noncoding RNA Cfast regulates cardiac fibrosis. Mol Ther Nucleic Acids 23: 377-392, 2020.

14. Statello L, Guo CJ, Chen LL and Huarte M: Gene regulation by long non-coding RNAs and its biological functions. Nat Rev Mol Cell Biol 22: 96-118, 2021.

15. Su J, Fang M, Tian B, Luo J, Jin C, Wang X, Ning Z and Li X: Atorvastatin protects cardiac progenitor cells from hypoxia-induced cell growth inhibition via MEG3/miR-22/ HMGB1 pathway. Acta Biochim Biophys Sin (Shanghai) 50: 1257-1265, 2018.

16. Li X, Zhao J, Geng J, Chen F, Wei Z, Liu C, Zhang X, Li Q, Zhang J, Gao L, et al: Long non-coding RNA MEG3 knockdown attenuates endoplasmic reticulum stress-mediated apoptosis by targeting p53 following myocardial infarction. J Cell Mol Med 23: 8369-8380, 2019.

17. Wu H, Zhao ZA, Liu J, Hao K, Yu Y, Han X, Li J, Wang Y, Lei W, Dong N, et al: Long noncoding RNA Meg3 regulates cardiomyocyte apoptosis in myocardial infarction. Gene Ther 25: 511-523, 2018.

18. Gong L, Xu H, Chang H, Tong Y, Zhang $\mathrm{T}$ and Guo G: Knockdown of long non-coding RNA MEG3 protects H9c2 cells from hypoxia-induced injury by targeting microRNA-183. J Cell Biochem 119: 1429-1440, 2018.

19. Qu C, Liu X, Ye T, Wang L, Liu S, Zhou X, Wu G, Lin J, Shi S and Yang B: miR-216a exacerbates TGF- $\beta$-induced myofibroblast transdifferentiation via PTEN/AKT signaling. Mol Med Rep 19: 5345-5352, 2019.

20. Livak KJ and Schmittgen TD: Analysis of relative gene expression data using real-time quantitative PCR and the 2(-Delta Delta C(T)) method. Methods 25: 402-408, 2001.

21. Piccoli MT, Gupta SK, Viereck J, Foinquinos A, Samolovac S, Kramer FL, Garg A, Remke J, Zimmer K, Batkai S and Thum T: Inhibition of the cardiac fibroblast-enriched lncRNA Meg3 prevents cardiac fibrosis and diastolic dysfunction. Circ Res 121: 575-583, 2017. 
22. Purnomo Y, Piccart Y, Coenen T, Prihadi JS and Lijnen PJ: Oxidative stress and transforming growth factor- $\beta 1$-induced cardiac fibrosis. Cardiovasc Hematol Disord Drug Targets 13: 165-172, 2013.

23. Wu J, Xia S, Kalionis B, Wan W and Sun T: The role of oxidative stress and inflammation in cardiovascular aging. Biomed Res Int 2014: 615312, 2014.

24. Tsutsui H, Kinugawa S and Matsushima S: Mitochondrial oxidative stress and dysfunction in myocardial remodelling. Cardiovasc Res 81: 449-456, 2009.

25. Zhang Y, Dees C, Beyer C, Lin NY, Distler A, Zerr P, Palumbo K, Susok L, Kreuter A, Distler O, et al: Inhibition of casein kinase II reduces TGF $\beta$ induced fibroblast activation and ameliorates experimental fibrosis. Ann Rheum Dis 74: 936-943, 2015.

26. Milara J, Hernandez G, Ballester B, Morell A, Roger I, Montero P, Escrivá J, Lloris JM, Molina-Molina M, Morcillo E and Cortijo J: The JAK2 pathway is activated in idiopathic pulmonary fibrosis. Respir Res 19: 24, 2018.

27. Yusuf S, Joseph P, Rangarajan S, Islam S, Mente A, Hystad P, Brauer M, Kutty VR, Gupta R, Wielgosz A, et al: Modifiable risk factors, cardiovascular disease, and mortality in 155722 individuals from 21 high-income, middle-income, and low-income countries (PURE): A prospective cohort study. Lancet 395: 795-808, 2020

28. El-Baz FK, Aly HF and Abd-Alla HI: The ameliorating effect of carotenoid rich fraction extracted from Dunaliella salina microalga against inflammation-associated cardiac dysfunction in obese rats. Toxicol Rep 7: 118-124, 2019.

29. Xu H, Shen Y, Liang C, Wang H, Huang J, Xue P and Luo M: Inhibition of the mevalonate pathway improves myocardial fibrosis. Exp Ther Med 21: 224, 2021.

30. Hu F, Li M, Han F, Zhang Q, Zeng Y, Zhang W and Cheng X: Role of TRPM7 in cardiac fibrosis: A potential therapeutic target (review). Exp Ther Med 21: 173, 2021.

31. Nasir K, Tsai M, Rosen BD, Fernandes V, Bluemke DA, Folsom AR and Lima JA: Elevated homocysteine is associated with reduced regional left ventricular function: The multi-ethnic study of atherosclerosis. Circulation 115: 180-187, 2007.

32. Stampfer MJ, Malinow MR, Willett WC, Newcomer LM, Upson B, Ullmann D, Tishler PV and Hennekens CH: A prospective study of plasma homocyst(e)ine and risk of myocardial infarction in US physicians. JAMA 268: 877-881, 1992.

33. Zhao Q, Song W, Huang J, Wang D and Xu C: Metformin decreased myocardial fibrosis and apoptosis in hyperhomocysteinemia-induced cardiac hypertrophy. Curr Res Transl Med 69: 103270, 2021.

34. Perbellini F, Watson SA, Scigliano M, Alayoubi S, Tkach S, Bardi I, Quaife N, Kane C, Dufton NP, Simon A, et al Investigation of cardiac fibroblasts using myocardial slices. Cardiovasc Res 114: 77-89, 2018

35. Moghadaszadeh B and Beggs AH: Selenoproteins and their impact on human health through diverse physiological pathways. Physiology (Bethesda) 21: 307-315, 2006.

36. Papp LV, Lu J, Holmgren A and Khanna KK: From selenium to selenoproteins: Synthesis, identity, and their role in human health. Antioxid Redox Signal 9: 775-806, 2007.

37. Rayman MP: Selenium and human health. Lancet 379: 1256-1268, 2012.

38. Gao Y, Feng HC, Walder K, Bolton K, Sunderland T, Bishara N, Quick M, Kantham L and Collier GR: Regulation of the selenoprotein SelS by glucose deprivation and endoplasmic reticulum stress-SelS is a novel glucose-regulated protein. FEBS Lett 563: $185-190,2004$
39. Bomer N, Grote Beverborg N, Hoes MF, Streng KW, Vermeer M, Dokter MM, IJmker J, Anker SD, Cleland JGF, Hillege HL, et al: Selenium and outcome in heart failure. Eur J Heart Fail 22: $1415-1423,2020$

40. Liu H, Xu H and Huang K: Selenium in the prevention of atherosclerosis and its underlying mechanisms. Metallomics 9: 21-37, 2017.

41. Chen Y, Zhang Z, Zhu D, Zhao W and Li F: Long non-coding RNA MEG3 serves as a ceRNA for microRNA-145 to induce apoptosis of AC16 cardiomyocytes under high glucose condition. Biosci Rep 39: BSR20190444, 2019.

42. Zou L, Ma X, Lin S, Wu B, Chen Y and Peng C: Long noncoding RNA-MEG3 contributes to myocardial ischemia-reperfusion injury through suppression of miR-7-5p expression. Biosci Rep 39: BSR20190210, 2019.

43. Uchida S: Besides imprinting: Meg3 regulates cardiac remodeling in cardiac hypertrophy. Circ res 121: 486-487, 2017.

44. Suzuki S, Tanaka K and Suzuki N: Ambivalent aspects of interleukin-6 in cerebral ischemia: Inflammatory versus neurotrophic aspects. J Cereb Blood Flow Metab 29: 464-479, 2009.

45. Bujak M, Dobaczewski M, Chatila K, Mendoza LH, Li N, Reddy A and Frangogiannis NG: Interleukin-1 receptor type I signaling critically regulates infarct healing and cardiac remodeling. Am J Pathol 173: 57-67, 2008.

46. Takahashi K, Fukushima S, Yamahara K, Yashiro K, Shintani Y, Coppen SR, Salem HK, Brouilette SW, Yacoub MH and Suzuki K: Modulated inflammation by injection of high-mobility group box 1 recovers post-infarction chronically failing heart. Circulation 118 (Suppl 14): S106-S114, 2008.

47. Zhang C, Deng Y, Lei Y, Zhao J, Wei W and Li Y: Effects of selenium on myocardial apoptosis by modifying the activity of mitochondrial STAT3 and regulating potassium channel expression. Exp Ther Med 14: 2201-2205, 2017.

48. Kucinski I, Dinan M, Kolahgar G and Piddini E: Chronic activation of JNK JAK/STAT and oxidative stress signalling causes the loser cell status. Nat Commun 8: 136, 2017.

49. Magaye RR, Savira F, Hua Y, Xiong X, Huang L, Reid C, Flynn B, Kaye D, Liew D and Wang BH: Exogenous dihydrosphingosine 1 phosphate mediates collagen synthesis in cardiac fibroblasts through JAK/STAT signalling and regulation of TIMP1. Cell Signal 72: 109629, 2020.

50. Su SA, Yang D, Wu Y, Xie Y, Zhu W, Cai Z, Shen J, Fu Z, Wang Y, Jia L, et al: EphrinB2 regulates cardiac fibrosis through modulating the interaction of Stat 3 and TGF- $\beta / \mathrm{Smad} 3$ signaling. Circ Res 121: 617-627, 2017.

51. Liu M, Li Y, Liang B, Li Z, Jiang Z, Chu C and Yang J: Hydrogen sulfide attenuates myocardial fibrosis in diabetic rats through the JAK/STAT signaling pathway. Int J Mol Med 41: 1867-1876, 2018.

This work is licensed under a Creative Commons Attribution-NonCommercial-NoDerivatives 4.0 International (CC BY-NC-ND 4.0) License. 